\title{
Flexible bronchoscopy in pulmonary diseases in children with congenital cardiovascular abnormalities
}

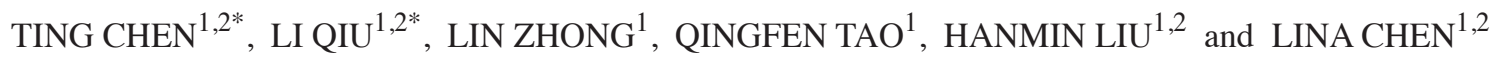 \\ ${ }^{1}$ Department of Pediatrics; ${ }^{2}$ Key Laboratory of Obstetric and Gynecologic and Pediatric Diseases and Birth Defects of \\ Ministry Education, West China Second University Hospital, Sichuan University, Chengdu, Sichuan 610041, P.R. China
}

Received October 12, 2017; Accepted March 5, 2018

DOI: $10.3892 /$ etm.2018.6113

\begin{abstract}
There is a lack of data describing the role of flexible bronchoscopy (FB) in evaluating pulmonary diseases in children with congenital cardiovascular abnormalities in China. Determining whether those children suffer from respiratory complications may be useful for future preoperative planning and family counseling. The present study aimed to investigate the features and FB-associated findings with respect to pulmonary diseases in children with congenital cardiovascular abnormalities. The role of FB in guiding the treatment and safety of procedures was also evaluated. A cohort of 57 children with congenital cardiovascular abnormalities underwent FB for pulmonary diseases between November 2013 and June 2015. The demographics, bronchoscopy diagnoses, cellular analysis and microbiology of bronchoalveolar lavage fluid (BALF), and the clinically valuable contributions and side-effects of FB were analyzed retrospectively. The bronchoscopies were performed in patients with a median age of 4 months (range, 9 days- 9 years) and 9 patients were intubated and mechanically ventilated. The most common types of congenital cardiovascular abnormalities were atrial septal defect, ventricular septal defect and patent ductus arteriosus. External compression of airways, tracheobronchomalacia, laryngomalacia, and airway narrowing were the most common airway abnormalities revealed by FB. BALF cellular analysis revealed an elevated total cell count and neutrophil percentage; $19.2 \%$ specimens harbored an etiological agent. FB findings contributed towards the clinical management of $26.3 \%$ patients. The complications of FB were mild and transient. In the present study, the majority of patients with cardiovascular abnormalities exhibited other airway disorders.
\end{abstract}

Correspondence to: Dr Lina Chen, Department of Pediatrics, West China Second University Hospital, Sichuan University, 20 Renmin South Road, 3rd Section, Chengdu, Sichuan 610041, P.R. China E-mail: chenln66@163.com

*Contributed equally

Key words: flexible bronchoscopy, children, cardiovascular abnormalities, pneumonia, airway diseases, bronchoalveolar lavage
The present findings suggest that FB was a useful and safe tool in the evaluation of this specific group of children.

\section{Introduction}

Congenital cardiovascular abnormalities and respiratory diseases are two tightly linked entities. Although respiratory diseases, particularly infections, are common in children, occasionally, they may be life-threatening if those suffering from congenital cardiovascular abnormalities are affected (1). Furthermore, $\geq 3 \%$ of children with cardiac disease also exhibit airway issues, including tracheobronchomalacia and airway narrowing, which are known to be latent causes and aggravating factors of persistent and recurrent lower respiratory infections $(2,3)$. A rapid and accurate diagnosis is essential for the initiation of appropriate treatment in children presenting both pulmonary diseases and congenital cardiovascular abnormalities (1). Determining whether these children suffer from respiratory complications such as airway anomalies may prove valuable for future preoperative planning and counseling of their families (4).

Flexible bronchoscopy (FB) is a crucial and safe technique for the evaluation of pulmonary diseases, allowing assessment of anatomy, dynamics of airways, and determination of pathogens via bronchoalveolar lavage (BAL) fluid (BALF) examinations $(3,5)$. With respect to certain airway abnormalities, such as tracheomalacia and cartilage rings, FB persists as the 'gold standard' method of diagnosis $(6,7)$. In addition, previous studies have demonstrated its potential in evaluating patients with diseases of cardiovascular and gastrointestinal systems $(1,4,6,7)$.

Although FB has been performed in China for over 20 years, sufficient data describing the role of FB in evaluating lung and airway diseases in children with congenital cardiovascular abnormalities remains scarce. In 2009, a study solely focused on airway compression complicated with excessive vessel abnormalities was performed (7). Another study summarized the bronchoscopy-associated diagnoses in children with the congenital cardiac disease without an in-depth analysis (4).

The present study aimed to investigate the features of children with congenital cardiovascular abnormalities who underwent FB due to pulmonary problems. The bronchoscopy findings, and cellular and culture results are presented, and were analyzed to assess the role of FB in guiding preoperative planning, and evaluate the safety of the procedures in those children. 


\section{Materials and methods}

Patients. The present study was carried out at West China Second University Hospital, Sichuan University (Chengdu, China). A review of medical records of all pediatric patients with congenital cardiovascular abnormalities who underwent FB between November 1,2013 and June 31,2015 was conducted. A total of 57 patients (male:female, 29:28; age, 9 days-9 years) were included. In order to fulfill the inclusion criteria, patients should have suffered from confirmed congenital cardiovascular abnormalities and pulmonary diseases (as indicated in Table I and Fig. 1) that were indicated for FB. The exclusion criteria were as follows: Patients with incomplete records and those whose parents or guardians refused to allow participation in the study.

Cardiovascular abnormalities were evaluated via transthoracic echocardiography (GE Vivid E9 Cardiovascular Ultrasound System; GE Healthcare, Chicago, IL, USA). Magnetic resonance angiography (MRA; Achieva 1.5T Magnetic Resonance Diagnostic Device; Philips Medical Systems B.V., Eindhoven, The Netherlands) indicated the suspected vessel abnormalities. Cardiac diagnoses were made by pediatric cardiologists based on clinical, transthoracic echocardiography, or MRA findings.

The Institutional Review Board/Ethics Committee affiliated with West China Second University Hospital, Sichuan University approved the current study, which was performed in accordance with the ethical standards of the Declaration of Helsinki. Parents or guardians of all patients provided written, informed consent prior to the procedures.

Indications for $F B$. All patients were hospitalized and exhibited pneumonia. The indications for FB were as follows: Failure to extubate in 6 patients, searching for pathogens in 15 patients, consolidation in 14 patients, wheezing or suspicion of airway anomaly in 15 patients, and persistent atelectasis or hyperinflation in 7 patients (Fig. 1). Failure to extubate was defined as two or more failed attempts to extubate or failing to meet the criteria for extubating (2).

Procedure of FB and bronchoalveolar lavage. All patients were evaluated by a pediatric pulmonologist.FB was performed at the West China Second University Hospital, Sichuan University by three experienced attending pediatric pulmonologists using a bronchoscope (P260F or XP260F; Olympus Corporation, Tokyo, Japan) in the Bronchoscopy Room, apart from 8 patients who were supported by mechanical ventilation, who underwent FB in the pediatric intensive care unit. Procedures were performed in compliance with the Chinese Guide To Pediatric Bronchoscopy (8). Patients were sedated with intravenous midazolam (Jiangsu Nhwa Pharmaceutical Co., Ltd., Jiangsu, China; 0.1-0.3 mg/kg). Topical anesthesia with $1 \%$ lidocaine $(5-7 \mathrm{mg} / \mathrm{kg}$ ) was administered via the nose, vocal cords, trachea, and bronchus. The transnasal approach was used in all patients who were not intubated, whereas the endotracheal approach was utilized in those who were intubated and mechanically ventilated (8).

During the procedure, vital signs and pulse oxygen saturation of all patients were continuously monitored and recorded as continuous oxygen was delivered. BAL was performed in the abnormal region identified during physical examination, radiography or chest computed tomography (CT) scan, or in the right middle or left lingual lobe when no localization signs were present. Normal saline solution ( $1 \mathrm{ml} / \mathrm{kg}$, three times) was instilled followed by immediate aspiration of the fluid into a sterile container. BALF was further subjected to cellular analysis, Gram- and acid-fast staining, bacterial culture, fungal smear, and culture as previously described $(9,10)$.

Data collection and outcome measures. Data was obtained by reviewing the medical records, managed with Microsoft Office Excel 2016 (Microsoft Corporation, Redmond, WA, USA) and described as frequencies. The demographics, results of radiography and transthoracic echocardiography, bronchoscopy diagnoses, results of the cellular analysis, and microbiology of BALF were recorded. Bronchoscopy diagnoses were made by direct observation of airways by at least two of the aforementioned experienced attending pediatric pulmonologists. Disagreements between observers were resolved by consensus. Clinically valuable contributions (defined as bronchoscopy diagnosis leading to altered antimicrobial treatment, assistance in preoperative planning and resolution of atelectasis) were analyzed. The putative side effects of FB such as fever, hemorrhage, arrhythmia, and sudden death were also recorded meticulously.

\section{Results}

Characteristics of patients. A total of 57 patients with congenital cardiovascular abnormalities combined with pulmonary diseases were enrolled in the present study and a bronchoscopy was performed on each patient during the study period. The cohort comprised of 29 male and 28 female patients, and the median age was 4 months (range, 9 days- 9 years). The number of patients aged $<29$ days, 29 days- 1 year, and $>1$ year were 4 (7.0\%), $43(75.4 \%)$, and $10(17.6 \%)$, respectively. A total of 9 patients $(15.8 \%)$ who were under mechanical ventilation underwent FB through the endotracheal tube, whereas the other 48 patients $(84.2 \%)$ were examined via the transnasal approach. The most common type of congenital cardiovascular abnormality was atrial septal defect (ASD; 24/57, 42.2\%), followed by ventricular septal defect (VSD; 11/57, 19.3\%) and patent ductus arteriosus (PDA; 8/57, 14.0\%). In addition, 2 cases with Tetralogy of Fallot (TOF; 2/57, 3.5\%) were enrolled. Other types of cardiovascular disorders consisted of 1 case of stenosis of aortic arch, 1 of dilated vena coronaria and a patent foramen ovale, 2 of pulmonary slings and ASD, 2 of total anomalous pulmonary venous connection (TAPVC), 1 of complete vascular ring, and 1 of VSD combined with ASD and PDA. A total of 2 patients with TAPVC and 1 with VSD plus ASD underwent open-chest surgery and interventional operation, respectively, prior to FB. The characteristics of patients who underwent FB according to the cardiovascular abnormalities are presented in Table I.

Bronchoscopy findings. The primary findings of FB were signs of infection $(57 / 57,100 \%)$ such as increased secretion, mucosal hyperemia, and edema of airways, which coincided with that of pneumonia. In addition, the second most common findings were disorders associated with airway abnormalities, 
Table I. Characteristics of patients undergoing bronchoscopy according to cardiovascular disorders.

\begin{tabular}{|c|c|c|c|c|c|}
\hline $\begin{array}{l}\text { Type of } \\
\text { cardiovascular disorders }\end{array}$ & $\begin{array}{l}\text { Patients, } \\
\mathrm{n}(\%)\end{array}$ & $\begin{array}{c}\text { Median } \\
\text { age, years }\end{array}$ & $\begin{array}{l}\text { Sex ratio, } \\
\text { male/female }\end{array}$ & $\begin{array}{c}\text { Body } \\
\text { weight, kg }\end{array}$ & $\begin{array}{l}\text { Mechanically } \\
\text { ventilated, } \mathrm{n}\end{array}$ \\
\hline ASD & $24(42.2)$ & 0.46 & $14 / 10$ & $6.23 \pm 2.71$ & 3 \\
\hline VSD & $11(19.3)$ & 0.25 & $5 / 6$ & $4.65 \pm 1.73$ & 2 \\
\hline PDA & $8(14.0)$ & 0.28 & $5 / 3$ & $5.11 \pm 2.23$ & 1 \\
\hline VSD+ASD & $4(7.0)$ & 0.17 & $0 / 4$ & $9.35 \pm 11.12$ & 1 \\
\hline TOF & $2(3.5)$ & 0.42 & $0 / 2$ & $6.55 \pm 0.21$ & 0 \\
\hline Other & $8(14.0)$ & 0.79 & $3 / 5$ & $7.73 \pm 3.22$ & 2 \\
\hline Total & $57(100.0)$ & 0.33 & $29 / 28$ & $6.21 \pm 3.71$ & 9 \\
\hline
\end{tabular}

Body weight data are presented as the mean \pm standard deviation. ASD, atrial septal defect; VSD, ventricular septal defect; PDA, patent ductus arteriosus; TOF, tetralogy of Fallot.

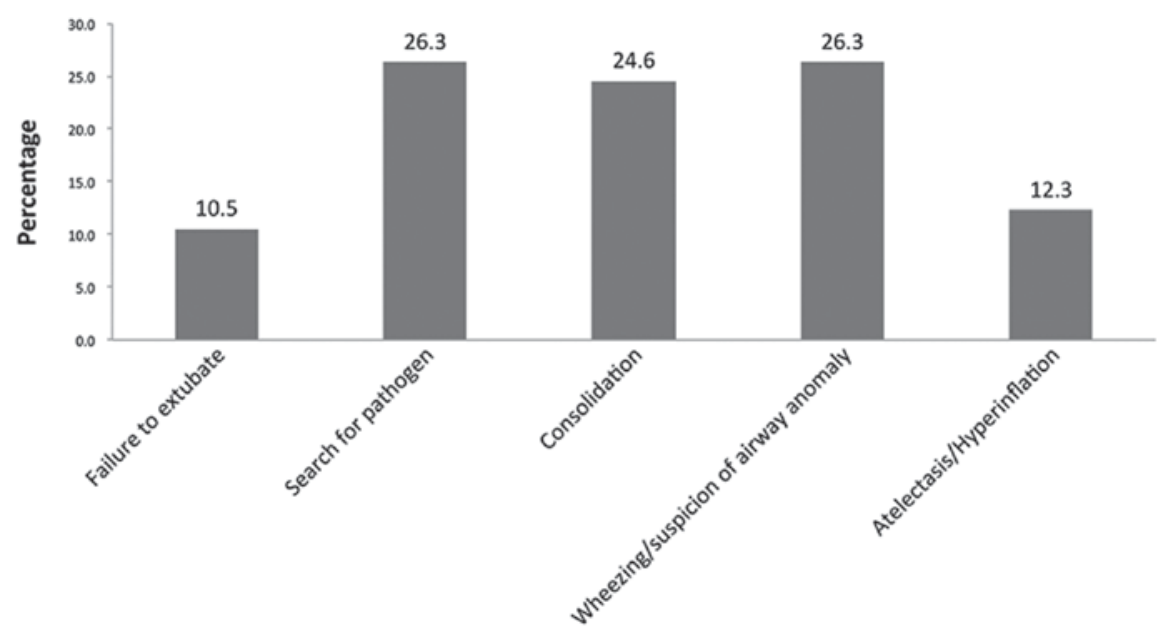

Figure 1. Indications for flexible bronchoscopy $(\mathrm{n}=57)$.

including 19 cases of external compression of airways (33.3\%), tracheobronchomalacia in 16 cases $(28.1 \%)$, and 12 cases of laryngomalacia and airway narrowing (21.1\%). A single case of mucosal bleeding was diagnosed in a 4-year-old Tibetan girl with pneumonia and ASD. Other diagnoses comprised of 4 cases presenting bridging bronchus, 4 cases of tracheal bronchus, and 1 case each of polyp of the vocal cord, complete tracheal rings, neoplasm in the trachea, neoplasm in the lingual root, and absence of the right upper lobe (Table II).

Among 57 patients, only 14 (24.6\%) presented bronchoscopy findings in accordance with the infection. Thus, 43/57 (75.4\%) patients exhibited $\geq 2$ bronchoscopy diagnoses, among which the infection combined with airway abnormality was most common (Fig. 2).

Furthermore, different types of cardiovascular abnormalities presented various airway abnormalities (Table III). Notably, the more complicated the patients' cardiovascular abnormalities were, the more complicated airway abnormalities they exhibited. Rare airway abnormalities, including bridging trachea and complete tracheal rings were present in patients with multiple congenital cardiovascular abnormalities. Common airway anomalies, including the external compression of airways, airway narrowing, tracheobronchonmalacia and laryngomalacia were commonly observed in patients with one cardiovascular anomaly such as ASD, VSD or PDA.

BALF assessments. BALF cellularity analysis was performed in 50 patients. A total of 7 patients were excluded as they were too young to tolerate repeated bronchoalveolar lavage, this meant the amount of BALF gathered was not enough for cellularity analysis and culture. The BALF cellularity analysis revealed an increased total cell count (mean, 1631.3 $\pm 497.2 \times 10^{6} / \mathrm{ml}$ ), and the median percentage of neutrophils and lymphocytes were 44.0 and $9.0 \%$, respectively. The increased total cell count and neutrophil percentage were in accordance with the infection in these patients.

Furthermore, 10/52 (19.2\%) BALF specimens exhibited a positive isolation of an etiological agent including 3 cases of Klebsiella pneumoniae, 2 cases of acid-producing Klebsiella and Bauman's Acinetobacter, and 1 case each of Staphylococcus aureus, Haemophilus influenzae, and Candida parapsilosis.

Clinically valuable contribution of FB. FB findings contributed towards the clinically valuable management of 15 patients (26.3\%). A total of 2 patients (3.5\%) were treated with 
Table II. Diagnosis of flexible bronchoscopy $(n=57)$.

\begin{tabular}{lc}
\hline Diagnosis & Patients, $\mathrm{n}(\%)$ \\
\hline Infection & $57(100)$ \\
External compression of airways & $19(33.3)$ \\
Tracheobronchomalacia & $16(28.1)$ \\
Laryngomalacia & $12(21.1)$ \\
Airway narrowing & $12(21.1)$ \\
Others & $13(22.8)$ \\
Bridging bronchus & $4(7.0)$ \\
Tracheal bronchus & $4(7.0)$ \\
Complete tracheal rings & $1(1.8)$ \\
Polyp of vocal cord & $1(1.8)$ \\
Neoplasm in trachea & $1(1.8)$ \\
Neoplasm in the lingual root & $1(1.8)$ \\
Absence of right upper lobe & $1(1.8)$ \\
Mucosal bleeding & $1(1.8)$ \\
\hline
\end{tabular}

Vancomycin (10 mg/kg every $6 \mathrm{~h})$, Piperacillin and Tazobactam (30 mg/kg every $8 \mathrm{~h}$ ) alternatively, following positive isolation of Staphylococcus aureus and Haemophilus influenzae from BALF. FB-based diagnoses assisted in the preoperative planning for 8 patients $(14.0 \%)$ including bridging bronchus in 4 patients (7.0 \%), complete tracheal rings in $1(1.8 \%)$, polyp of vocal cord in $1(1.8 \%)$, tracheal neoplasm in $1(1.8 \%)$, and neoplasm in the lingual root in $1(1.8 \%)$. Furthermore, atelectasis was resolved in 5 patients $(9.0 \%)$ following the FB procedure.

Complications. The procedure of FB was safe. The complications observed were mild and easily corrected, including fever following procedure in 1 patient and $(1.8 \%)$ mild desaturation (peripheral capillary oxygen saturation $<92 \%$ ) in 3 patients $(5.3 \%)$ that were resolved by supplemental oxygen. Mild bleeding was exhibited by 1 patient, which was ceased by administering 1-2 ml 1:10,000 epinephrine. The total incidence of complications was $8.8 \%$. There were 0 instances of mortality during or immediately following the FB.

\section{Discussion}

Children with congenital cardiovascular abnormalities are predisposed to respiratory diseases. As cardiovascular abnormalities are associated with airway disorders, occasionally, their respiratory symptoms are challenging to resolve. FB presents a diagnostic and therapeutic role in the evaluation of pulmonary diseases (2). In the present study, it was demonstrated that FB is a clinically useful and safe technique for the evaluation of pulmonary diseases in children with congenital cardiovascular abnormalities.

FB confirmed that $75.4 \%$ of all patients enrolled in the present study exhibited complications associated with airway disorders. The spectrum of airway diseases observed is similar to that described in previous studies $(1,11,12)$. Herein, the most common bronchoscopy diagnoses of airway abnormalities consisted of external compression of airways, tracheobronchomalacia, and airway narrowing, which are associated with cardiovascular abnormalities such as ASD, VSD, and PDA. The possible reasons why cardiovascular abnormalities are associated with airway disorders have been discussed elsewhere $(1,2,13,14)$. Notably, a number of rare airway abnormalities such as bridging bronchus, tracheal bronchus, and complete tracheal ring were also presented, for which surgical correction was suggested. Although certain diagnostic evaluations, such as MRI, are non-invasive and are able to display the structure of the tracheobronchial tree, the heart, and the ambient vessels, FB remains the technique of choice for the evaluation of the airways in the event of tracheomalacia, stenosis, complete tracheal rings, or aberrant bronchi (4). Furthermore, identification of these airway abnormalities is useful in assisting preoperative planning prior to cardiothoracic surgery. Surgeons may be more cautious in airway management prior to and following cardiothoracic surgery to decrease the incidence of airway-related complications, such as airway stenosis following intubation and tracheostomy (15).

Congenital cardiovascular abnormalities accompanied by airway disorders may result in various clinical manifestations, such as recurrent lower respiratory infections, consolidation, atelectasis, hyperinflation, and wheezing $(1,2)$. As mentioned in a previous study, the bronchoscopic procedure alone is only seldom curative (1). However, certain procedures during FB may initiate the relief of pulmonary disease $(1,2,5)$. For example, the airway mucin and sputum plug may be aspirated out, which is beneficial for the resolution of consolidation, atelectasis, and hyperinflation of the lungs. A previous study observed the effects of therapeutic BAL on refractory Mycoplasma pneumoniae and demonstrated that the early application of BAL shortened the duration of fever and hospitalization, improved laboratory indices, and promoted the resolution of atelectasis (16). In the present study, it was observed that atelectasis was resolved in 5 patients $(8.8 \%)$ following FB. In addition, the airway abnormalities, including narrowing and external compression of airways observed by FB, may help identify cardiovascular abnormalities. These pieces of evidence aid clinicians in revealing the underlying causes of symptoms, such as wheezing, to determine the diagnosis and initiate appropriate treatment as early as possible. In the present study, 2 patients exhibited airway narrowing; however, the chest $\mathrm{CT}$ failed to provide additional details. Following the procedures, MRA was arranged and pulmonary sling was confirmed as the cause of wheezing.

FB not only allows the assessment of airway anatomy and dynamics, but also provides information about infectious etiological agents. Although the initial choice of antimicrobial agents is empirical in the treatment of pulmonary infection, persistent efforts are made to identify the etiology by sample cultures $(5,17)$. Obtaining the sputum samples from children, particularly infants, is challenging as the majority are unable to cough out sputum voluntarily $(1,5)$. The treatment depends primarily on cultures from oropharyngeal specimens. FB and associated procedures (including BAL) allow the collection of lower respiratory specimens from patients from whom sputum samples are difficult to obtain. The cultures of bronchoscopy specimens provide a higher yield of organisms as compared to those from oropharyngeal specimens (17). In the present study, all patients exhibited an elevated total cell count and 
Table III. Bronchoscopic diagnosis of airway anomalies according to different type of cardiovascular disorders (n=57).

\begin{tabular}{lccccccc}
\hline $\begin{array}{l}\text { Type of } \\
\text { cardiovascular } \\
\text { disorders }\end{array}$ & $\begin{array}{c}\text { External } \\
\text { compression } \\
\text { of airways }\end{array}$ & Tracheobronchomalacia & Laryngomalacia & $\begin{array}{c}\text { Airway } \\
\text { narrowing }\end{array}$ & $\begin{array}{c}\text { Tracheal } \\
\text { bronchus }\end{array}$ & $\begin{array}{c}\text { Bridging } \\
\text { trachea }\end{array}$ & $\begin{array}{c}\text { tracheal } \\
\text { rings }\end{array}$ \\
\hline ASD & $4(7.0)$ & $3(5.3)$ & $6(10.5)$ & $5(8.8)$ & $0(0.0)$ & $0(0.0)$ & $0(0.0)$ \\
VSD & $5(8.8)$ & $4(7.1)$ & $2(3.5)$ & $1(1.8)$ & $1(1.8)$ & $0(0.0)$ & $0(0.0)$ \\
PDA & $2(3.5)$ & $4(7.1)$ & $2(3.5)$ & $3(5.3)$ & $0(0.0)$ & $1(1.8)$ & $0(0.0)$ \\
VSD+ASD & $1(1.8)$ & $1(1.8)$ & $1(1.8)$ & $1(1.8)$ & $3(5.3)$ & $0(0.0)$ & $0(0.0)$ \\
TOF & $2(3.5)$ & $1(1.8)$ & $0(0.0)$ & $0(0.0)$ & $0(0.0)$ & $0(0.0)$ & $0(0.0)$ \\
Other & $5(8.8)$ & $3(5.3)$ & $1(1.8)$ & $2(3.5)$ & $0(0.0)$ & $3(5.3)$ & $1(1.8)$ \\
Stenosis of aortic arch & $1(1.8)$ & $0(0.0)$ & $1(1.8)$ & $0(0.0)$ & $0(0.0)$ & $0(0.0)$ & $0(0.0)$ \\
Pulmonary sling+ASD & $2(3.5)$ & $2(3.5)$ & $0(0.0)$ & $0(0.0)$ & $0(0.0)$ & $2(3.5)$ & $0(0.0)$ \\
TAPVC & $1(1.8)$ & $0(0.0)$ & $0(0.0)$ & $1(1.8)$ & $0(0.0)$ & $0(0.0)$ & $0(0.0)$ \\
Complete vascular ring & $1(1.8)$ & $0(0.0)$ & $0(0.0)$ & $0(0.0)$ & $0(0.0)$ & $0(0.0)$ & $0(0.0)$ \\
VSD+ASD+PDA & $0(0.0)$ & $1(1.8)$ & $0(0.0)$ & $1(1.8)$ & $0(0.0)$ & $1(1.8)$ & $1(1.8)$ \\
Total & $19(33.3)$ & $16(28.1)$ & $12(21.1)$ & $12(21.1)$ & $4(7.1)$ & $4(7.1)$ & $1(1.8)$ \\
\hline
\end{tabular}

Data are presented as n (\%). ASD, atrial septal defect; VSD, ventricular septal defect; PDA, patent ductus arteriosus; TOF, tetralogy of Fallot; TAPVC, total anomalous pulmonary venous connection.

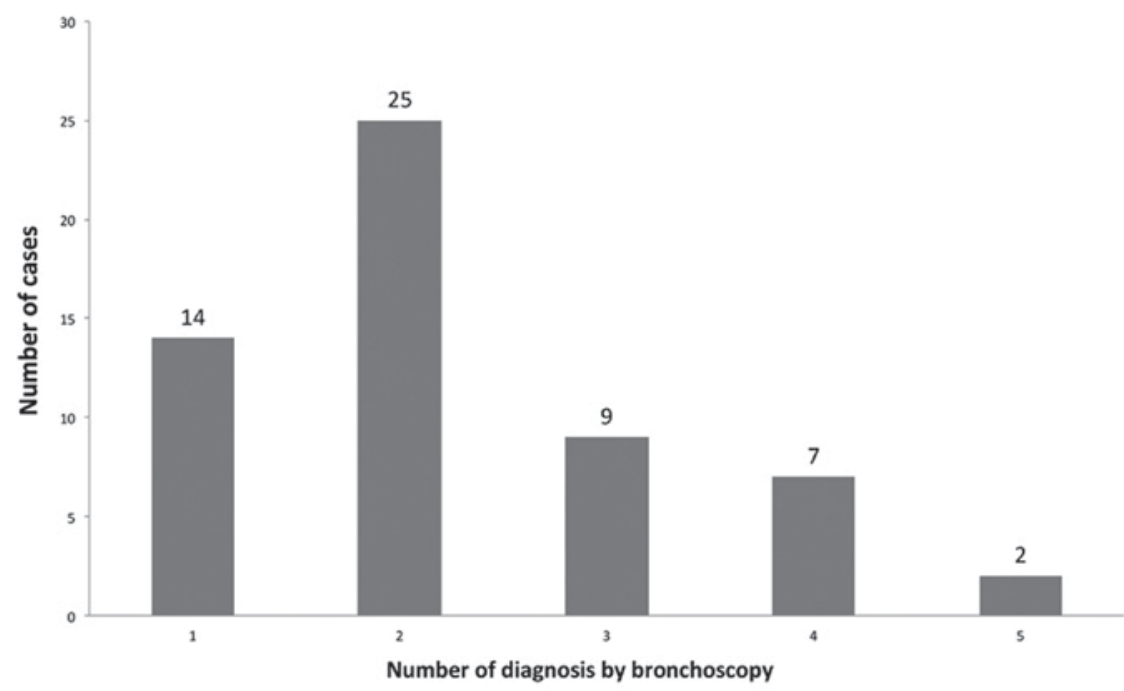

Figure 2. Distribution of diagnoses by bronchoscopy.

neutrophil percentage, which was similar to that of pneumonia. Furthermore, 10/52 patients exhibited positive BALF culture, which guided the alteration in the administration of antibiotics. The rate of pathogen recovery was similar to that in a previously published study (1). An accurate and immediate isolation of infectious agents is crucial for these patients, as the pulmonary infection may aggravate the original cardiac diseases leading to complications, such as cardiac failure, which may delay surgical interventions. Hence, FB helps to treat pneumonia and cardiac diseases.

FB is an invasive procedure and may have potentially severe side effects. Complications are associated with an individual patient's risk factors, medication used and ventilation technique. Typical complications include trauma of nose and airway, desaturation and hypoxemia, cough and bronchospasm, hemorrhage, pneumothorax and fever and infection (18). Thus, safety is a priority prior to performing FB, particularly in children. However, if patients and evaluated cautiously and the potential complications are considered, FB can be a safe technique. Although the majority of the present patients were young infants (the youngest was 9-days-old), a surprisingly low rate of complications were encountered: Only 5 patients exhibited mild complications, which were resolved immediately following supportive therapies. No mortality was recorded following $\mathrm{FB}$.

However, there are a number of limitations to the present study. First, the sample size is small, and the present study was a single-center study, which may influence the strength of the results. Second, the data were collected retrospectively, which may result in bias. Furthermore, analysis corresponding 
to the prognosis of the patients is absent, thereby necessitating further studies.

In conclusion, the present study supplements the literature describing the role of FB in the evaluation of pulmonary diseases in Chinese children with congenital cardiovascular abnormalities. In the present study, the majority of patients were complicated with other airway disorders. The data presented FB as a safe and effective tool for examining children with cardiovascular abnormalities. Therefore, FB may be considered as an initial tool in this specific group of children.

\section{Acknowledgments}

Not applicable.

\section{Funding}

The present study was partially supported by grants from the Science \& Technology department of Sichuan Province (grant no. 2015SZ0152) and the National Natural Science Foundation of China (grant no. 81200461).

\section{Availability of data and materials}

The datasets used and/or analyzed during the current study are available from the corresponding author on reasonable request.

\section{Authors' contributions}

TC was responsible for literature search, data collection, study design, analysis of data, manuscript preparation and review of manuscript. LQ was responsible for literature search, data collection, study design, manuscript preparation and review of manuscript. LZ was responsible for data collection, manuscript preparation and review of manuscript. QT was responsible for data collection and review of manuscript. HL was responsible for data collection and review of manuscript. LC was responsible for study design, literature search, data collection, analysis of data, manuscript preparation and review of manuscript.

\section{Ethics approval and consent to participate}

The present study has been approved by the Institutional Review Board/Ethics Committee of West China Second University Hospital, Sichuan University (Sichuan, China). Parents or guardians of all patients provided written, informed consent prior to the procedures.

\section{Consent for publication}

Parents or guardians of all patients provided written, informed consent for the publication of the associated data.

\section{Competing interests}

The authors declare that they have no competing interests.

\section{References}

1. Efrati O, Gonik U, Modan-Moses D, Bielorai B, Barak A, Vardi A, Paret G, Mishaly D and Toren A: The role of flexible fibreoptic bronchoscopy in evaluation of pulmonary diseases in children with congenital cardiac disease. Cardiol Young 17: 140-144, 2007.

2. Guillemaud JP, El-Hakim H, Richards S and Chauhan N: Airway pathologic abnormalities in symptomatic children with congenital cardiac and vascular disease. Arch Otolaryngol Head Neck Surg 133: 672-676, 2007.

3. Gokdemir Y, Cakir E, Kut A, Erdem E, Karadag B, Ersu R and Karakoc F: Bronchoscopic evaluation of unexplained recurrent and persistent pneumonia in children. J Paediatr Child Health 49: E204-E207, 2013.

4. Li JB, Chen XX, Hu XY, Cui YQ, Hu CM, Li FX and Chen JW: The application of flexible fiberoptic bronchoscope in perioperative management of the patients with congenital cardiac disease. Chin J Pediatr Surg 34: 173-175, 2013 (In Chinese).

5. Efrati O, Sadeh-Gornik U,Modan-Moses D, Barak A, Szeinberg A, Vardi A, Paret G, Toren A, Vilozni D and Yahav Y: Flexible bronchoscopy and bronchoalveolar lavage in pediatric patients with lung disease. Pediatr Crit Care Med 10: 80-84, 2009.

6. Nussbaum E: Pediatric fiberoptic bronchoscopy: Clinical experience with 2,836 bronchoscopies. Pediatr Crit Care Med 3: 171-176, 2002.

7. He SR, Sun YX, Liu YM, Zhuang J, Zhong J, Liang SX, Sun X and Lai JN: Airway obstruction caused by large blood vessel anomalies: Assessment by flexible bronchoscopy. Zhonghua Er Ke Za Zhi 47: 726-729, 2009 (In Chinese).

8. Pediatric Bronchoscopy Collaborative Group, The Subspecialty Group of Respiratory Diseases, The Society of Pediatrics, Chinese Medical Association. Guide to pediatric bronchoscopy (2009 edition). Zhonghua Er Ke Za Zhi 47: 740-744, 2009 (In Chinese).

9. Qun J, Zhou J and Yu Y: An official Chinese Thoracic Society clinical pratice guideline: The etiologic assessment of bronchoalveolar lavage in infectious lung disease. Chin J Tuberc Respir Dis 40: 578-583, 2017 (In Chinese).

10. Baselski VS and Wunderink RG: Bronchoscopic diagnosis of pneumonia. Clin Microbiol Rev 7: 533-558, 1994.

11. Lee SL, Cheung YF, Leung MP, Ng YK and Tsoi NS: Airway obstruction in children with congenital heart disease: Assessment by flexible bronchoscopy. Pediatr Pulmonol 34: 304-311, 2002.

12. Chapotte C, Monrigal JP, Pezard P, Jeudy C, Subayi JB, De Brux JL, Cottineau C and Granry JC: Airway compression in children due to congenital heart disease: Value of flexible fiberoptic bronchoscopic assessment. J Cardiothorac Vasc Anesth 12: 145-152, 1998.

13. Little AF, Phelan EM, Boldt DW and Brown TC: Paediatric tracheobronchomalacia and its assessment by tracheobronchography. Australas Radiol 40: 398-403, 1996.

14. Kussman BD, Geva T and McGowan FX: Cardiovascular causes of airway compression. Paediatr Anaesth 14: 60-74, 2004.

15. Wilson MN, Bergeron LM, Kakade A, Simon LM, Caspi J, Pettitt T and Kluka EA: Airway management following pediatric cardiothoracic surgery. Otolaryngol Head Neck 149: 621-627, 2013.

16. Zhang Y, Chen Y, Chen Z, Zhou Y, Sheng Y, Xu D and Wang Y: Effects of bronchoalveolar lavage on refractory Mycoplasma pneumoniae pneumonia. Respir Care 59: 1433-1439, 2014.

17. Jain K, Wainwright $C$ and Smyth AR: Bronchoscopy-guided antimicrobial therapy for cystic fibrosis. Cochrane Database Syst Rev: Cd009530, 2013.

18. Paradis TJ, Dixon J and Tieu BH: The role of bronchoscopy in the diagnosis of airway disease. J Thorac Dis 8: 3826-3837, 2016. 